\title{
THE INFLUENCE OF THE SHAPE OF DUST PARTICLES ON THE RATE OF PHAGOCYTOSIS IN VITRO
}

\author{
BY \\ A. LENGEROVÁ, V. J. LENGER, M. ESSLOVÁ, R. TUSCANY, and M. VOLFOVÁ \\ From the Department of Biophysics, Institute of Industrial Hygiene and Occupational Diseases, \\ Prague, Czechoslovakia
}

(RECEIVED FOR PUBLICATION APRIL 27, 1956)

Phagocytosis of the dust particles is one of the most striking phenomena observed in histological sections of lungs exposed to the effects of dusts. Therefore, a number of authors who tried to explain the mechanism of the origin of silicosis paid special attention to the question of phagocytosis.

Some of them were of the opinion (Fenn, 1922) that the rate of phagocytosis was indirectly proportional to the danger of various kinds of dusts. Fenn's results support this hypothesis as far as the effects of coal dust and quartz are concerned. On the other hand, Franks and Watt (1934) and Kasten (1939) found that these two kinds of dusts of biologically different efficiency were phagocytosed, roughly in the same degree, in an in vitro experiment. Further experiments by Policard (1948) have not led to general agreement on this problem. The lack of agreement may be caused partly by the variety of tissues used (embryonic lung tissue, spleen, suspensions of leucocytes), partly by differences in the state of the dusts, or perhaps to inadequacy in the methods of estimating the degree of phagocytosis.

Recent work shows that in addition to the effect of the chemical properties of the dust some physical properties may to some extent control its biological efficiency (King, Mohanty, Harrison, and Nagelschmidt, 1953). Some of the later work supports the hypothesis propounded by Fenn (1922) on the indirect relation between the " harmfulness" of dusts and the degree by which they are phagocytosed. Thus Jötten and Marwyck (1952) showed that protective dusts, which in animal experiments diminish the silicotic effectiveness of quartz, increase at the same time the efficiency by which quartz is phagocytosed in experiments in vitro. The knowledge of factors which may determine the rate of phagocytosis of quartz dusts appears therefore to be of great importance, and the shape of the quartz particles may be one of these determining factors.

\section{Material and Methods}

Our first objective was to ascertain the degree of dependence of the rate of phagocytosis on the shape of phagocytosed particles. The shape of particles was characterized by the relation of the minimum to the maximum dimension of their projection under the microscope, and each of these extreme dimensions had its own distribution in the given sample of dusts. An ideal arrangement of the experiment would have been one in which the rate of phagocytosis of particles for different ranges of ratios of extreme dimensions could have been ascertained. Since, however, such an experiment was not feasible, we proceeded in such a way that the particles were distributed into two groups: (1) the " long " particles where the length exceeds the width, and (2) the " round ones ", where both of these dimensions are approximately equal. Since such a determination can to a certain extent be considered subjective, a control was established by repeated classifications of particles from different sub-samples taken from the same principal sample. The ratios between the number of particles of the first and the second group in each sub-sample agreed well.

The study was carried out in cultures of macrophages derived from the spleen of 14-day chicken embryos cultivated in hanging drops. The degree of phagocytosis was evaluated with a phase-contrast microscope.

Reproducible doses of dusts were achieved by methods already described by Lengerová, Lenger, Esslová Tuscany, and Volfová (1956). The cultures were made on coverslips, dust being suspended in a diluted embryonic extract. Instead of normally hollow-ground slides, glasses with planparallel bottoms, which do not disturb observations through the phase-contrast microscope, were used. The distribution of the particle sizes was such that $99.4 \%$ did not exceed $5 \mu$.

For both shapes investigated the relation, $Z=\frac{N_{r}}{N_{l}}$, i.e., the relation between the number of the long and the round particles taken from a large number of particles $(15,000)$ was first estimated. With the experimental cultures, this relation was checked once more by counting 1,500 particles before inserting the cultures in the thermostat (t value). 
The methods of quantitative evaluation of phagocytosis in vitro consist in ascertaining the number of particles phagocytosed per phagocyte (Fenn, 1922; Jötten and Marwyck, 1952). In our experience, however, this procedure is unreliable, because, even when phase contrast microscopy is used, the number of phagocytosed particles of $\mathrm{SiO}_{2}$ cannot always be determined reliably; quartz particles within the cell gradually lose their sharp edges and after 24 hours the determination of the number of phagocytosed particles in the critical range up to $5 \mu$ is very unreliable. Thus an indirect procedure was used which is based on the determination of the decrease of the number of quartz particles in the zone of emigration of phagocytosing cells compared with the initial value at $t^{\circ}$. A possible objection to this procedure is that this decrease will include also those particles not phagocytosed but merely covered with macrophages. It was found, however, that with a suitable density of particles and macrophages this error was negligible. Moreover, the probability of the long or round particle being covered corresponds to the proportion of that particle in the sample, and, therefore, the fundamental relation $\mathrm{N}_{r} / \mathrm{N}_{l}$ is not changed.

With all cultures of macrophages the relation $\mathrm{N}_{r} / \mathrm{N}_{l}$ was estimated before inserting the cultures into the thermostat, and then during the incubation at four-hour intervals up to 24 hours. The evaluation of the number of particles was always carried out in the same sphere round the mother fragment, into which the macrophages emigrate, and that in the microscopic plane with the maximum number of macrophages. Some observations were made in which the cultures were followed throughout 24 hours of the experiment, a heated stage and phasecontrast equipment being used. Other cultures were fixed at different intervals with Carnoy's fluid, then mounted on Canada balsam, the decrease of particles being evaluated again by means of the phase-contrast microscope. For the different time periods the relation of $\mathrm{N}_{r} / \mathrm{N}_{l}$ was always based on examining an area in which there were about 500 macrophages.

\section{Results}

The time course of the relation of $\mathrm{N}_{r} / \mathrm{N}_{l}$ for quartz (curve 1) and for silicon carbide (curve 2) is shown in Fig. 1. During the first four hours of the experiment, when the emigration of macrophages is slight, the initial ratio remains practically unaltered and with $\mathrm{SiO}_{2}$ approximately equals 2 in favour of round particles. Between the fourth and the sixteenth hours the value $\mathrm{N}_{r} / \mathrm{N}_{l}$ gradually decreases. About the tenth hour the value begins to decrease until it is below 1. After 16 hours it again rises and at the end of the experiment, i.e., after 24 hours, the value 1 of $N_{r} / N_{l}$ is again reached.

For the dust of silicon carbide (curve 2, Fig. 1) the course of the relation $\mathrm{N}_{r} / \mathrm{N}_{l}$ is analogous but not so striking, i.e., with $\mathrm{SiC}$ the fundamental relation $\mathrm{N}_{r} / \mathrm{N}_{l}$ was just a little higher than 1 at the start and its decrease with time was less marked;

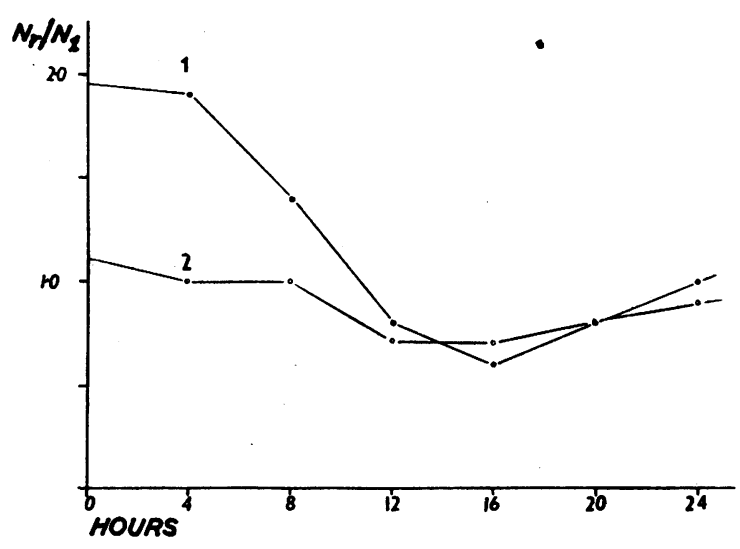

Fig. 1.-The time course of the relative values $N_{r} / N_{l} l$ throughout the duration of the experiment.

the same value as for $\mathrm{SiO}_{2}$, however, was nearly achieved, after which it began to return to 1 like quartz.

The real phagocytic activity of macrophages is not evident from Fig. 1, and that is why in Fig. 2 the decrease of an absolute number of $\mathrm{SiO}_{2}$ particles is shown in the mean field of view during the 24hours experiment, which is the result of the phagocytic activity of macrophages. Curve 1 refers to all $\mathrm{SiO}_{2}$ particles irrespective of shape distribution, curve 2 to the round particles, and curve 3 to the long ones. Fig. 3 demonstrates the velocity of phagocytosis of quartz particles as a function of time. Based on the experimental values, the velocity,

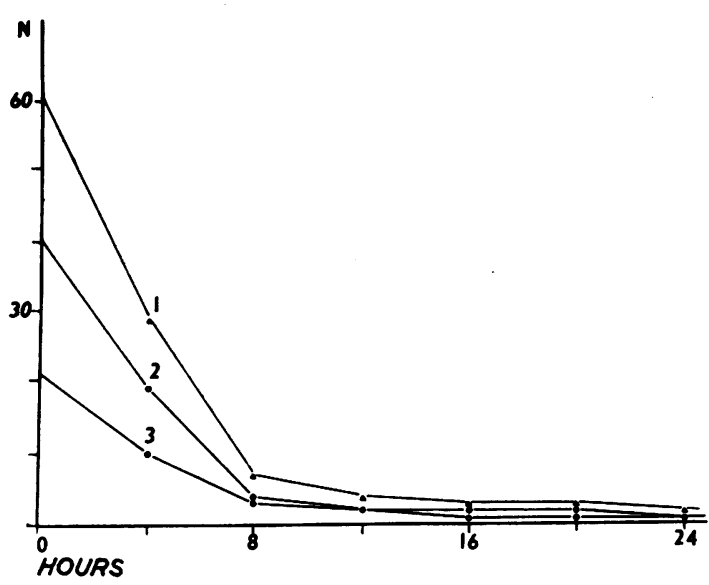

FIG. 2.-The decrease of an absolute number of quartz particles in the mean field of view during 24 hours of the experiment. Curve 1 represents $\mathrm{SiO}_{2}$ particles irrespective of shape distribution, curve 2 round particles, and curve 3 long particles. 


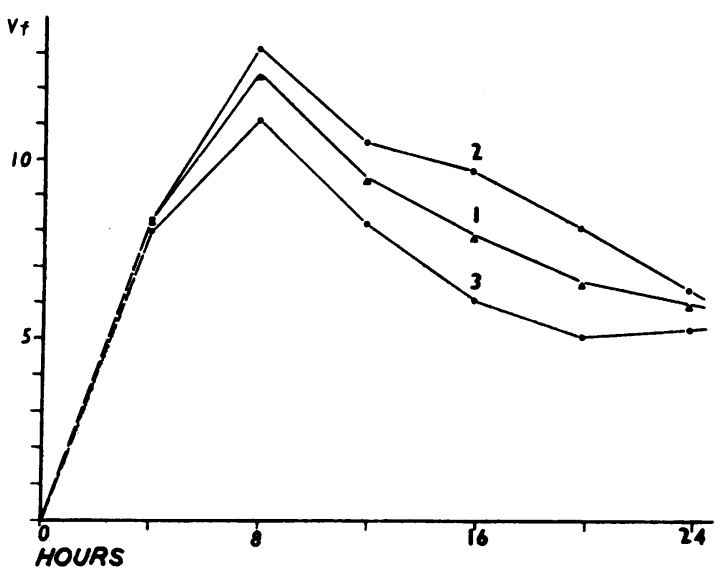

FIG. 3.-The velocity (Vf) of phagocytosis of quartz particles as a function of time ( $t$ in hours).

$\mathrm{V}_{f}$, is calculated from the relation given by Fenn (1921)

$$
V_{f}=(1 / t) \log \frac{A}{A-X}
$$

where $\mathbf{A}=$ the number of particles in the field of view, $\mathbf{X}=$ the number of phagocytosed particles, $\mathrm{t}=$ time.

It is quite evident that the velocity of phagocytosis of the round particles (curve 2) is higher than that of the long ones (curve 3 ), both reaching the maximum about the eighth hour of the experiment when the decrease occurs, and the values are almost the same for the remaining time, i.e., up to 24 hours. With $\mathrm{SiC}$ the ratios are similar. The decrease of the velocity of phagocytosis (Fig. 3) agrees in time with the decrease of an absolute number of particles in the field of view (Fig. 2), which points to the fact that the cause of the decrease of the phagocytic velocity is due to the decrease of the probability of a collision between a macrophage and a particle. In an attempt to interpret the curves obtained in Fig. 1 we have started from this premise. If the shape factor had no influence on the rate of phagocytosis of dust particles, the probability of phagocytosis of different extreme types of shape of particle would be proportional only to their presence in the sample of dust. Thus an absolute number of particles would decrease with the course of the experiment but the relative presence of both types of particle would remain constant. The course of the value $N_{r} / N_{l}$ would then be expressed by a straight line parallel to the axis $x$. In fact, the value $N_{r} / N_{l}$ decreases till under the value 1 , which means that with time the round particles decrease proportionately more rapidly than the long particles.
While at the beginning of the experiment there were twice as many round quartz particles as long ones, after 16 hours there was a larger number of long particles than round ones in the area of emigrated macrophages. Thus the round particles were phagocytosed preferentially. If we accept this opinion the fact that the increase of the value $N_{r} / N_{l}$ (Fig. 1) recurs after 16 hours of the experiment seems to be striking; this increase does not exceed the value 1 . Nevertheless, it seems to point to the fact that between the sixteenth and twenty-fourth hours it is again the long particles which are phagocytosed more effectively. We believe that this phenomenon may be explained by the majority of dust particles in the zone having been phagocytosed (Fig. 4) so that the error of estimating the ratio is large (Fig. 2).

We consider, therefore, that the first part of the curve in Fig. 2, proving the selective phagocytosis of round particles, is of great importance. This phenomenon cannot be explained to our satisfaction by any of the existing theories of phagocytosis. The influence of the shape could be important if phagocytosis of $\mathrm{SiO}_{2}$ particles consisted of a chemctactic. action of impurities adsorbed on the surface, which corresponds to McCutcheon's hypothesis (1942). The surface of the long particle is, roughly, $50 \%$

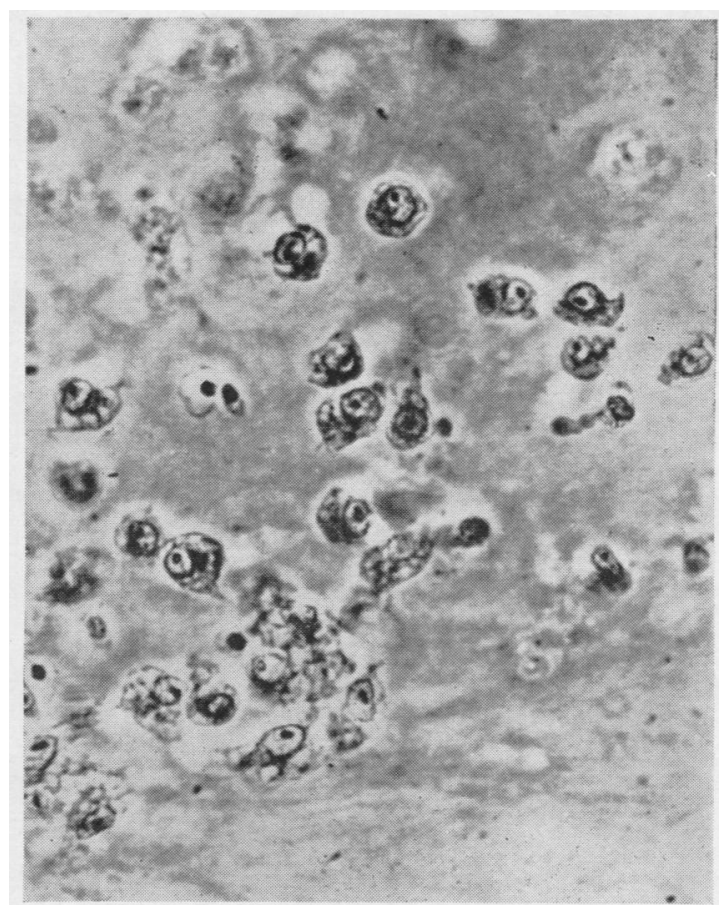

FIG. 4.-Photomicrograph of silicon carbide particles after 24 hours of the experiment. Most of the dust particles are already phagocytosed by the phagocytosing elements. 
greater than that of the round particle for the same volume, and consequently the long particle carries a greater quantity of absorbed impurities. As our experiments show that the long particles are substantially phagocytosed more slowly than the round ones, we should have to suppose (McCutcheon's hypothesis, 1942) that the activity of the adsorbed impurities is chemotactically negative to the phagocytosing elements.

It is also interesting to consider the influence of shape in relation to the findings of King and Nagelschmidt (1954) that the solubility of silicic acid represents the function of the specific surface and that the greater the surface the greater the fibroplastic effect. In this case, the long particles would again represent the particles of greater specific surface, which would therefore release more silicic acid and show a greater biological activity. In summary, it would mean that the particles of a certain shape might be biologically more active and at the same time be less readily phagocytosed, and so, according to the views of Fenn (1922), Lauche (1931), - Jötten and Marwyck (1952), and others, more harmful. Further experiments are necessary to prove this hypothesis when estimating the harmfulness of different dusts chemically identical.

\section{Summary}

The rate of phagocytosis of two shapes of dust particles-" long" and " round"-was studied in an investigation in vitro in cultures of macrophages from the spleen of chicken embryos. The round particles were phagocytosed preferentially. This result is discussed from the standpoint of existing theories of phagocytosis as well as from that of the relation between the rate of phagocytosis and the harmfulness of different kinds of dusts.

\section{REFERENCES}

Fenn, W. O. (1921). J. gen. Physiol., 3, 439, 465, 575. 1922). Cited by Jötten, K. W., and Marwyck, C. van (1952). Ibid., 4, 331.

Franks, W. R., and Watt, A. J. (1934). Trans. roy. Soc. Can., Sect. V, 3 rd ser., 28, 43.

Jötten, K. W., and Marwyck, C. van (1952). British Journal of Industrial Medicine, 9, 173.

Kasten, W. (1939). Arch. Gewerbepath. Gewerbehyg., 9, 337.

King, E. J., and Nagelschmidt, G. (1954). In Die Staublungenerkrankungen, vol. 2, ed. Jötten, K. W., Klosterkötter, W., and Pfefferkorn, G., p. 84. Steinkopff, Darmstadt. Proceedings of the second international conference on dust diseases of the

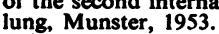

-, Mohanty, G. P., Harrison, C. V., and Nagelschmidt, G. (1953). British Journai of Industrial Medicine. 10, 9, 76.

Lauche, (1931). Verhandl. dtsch. path. Ges., 26, 107.

Lengerová, A., Lenger, V. J., Esslovà, M., Tuscany, R., and Volfová, M. (1956). Arch. Gewerbepath. Gewerbehyg., 14, 311.

McCutcheon, M. (1942). Arch. Path. (Chicago), 34, 167.

Policard, A. (1948). Experientia (Basel), 4, 113. 